\title{
Messung der Zyklotronfrequenz freier Elektronen im Vierpolkäfig
}

\author{
G. GRÄFF und E. KLEMPT
}

Physikalisches Institut der Universität Bonn

(Z. Naturforschg. 22 a, 1960-1962 [1967]; eingegangen am 2. August 1967)

\begin{abstract}
Elektrons were stored for several minutes in a homogeneous magnetic field and an electrical quadrupol field $V=\left(U_{0} / R^{2}\right) \cdot\left(x^{2}+y^{2}-2 z^{2}\right)$. The solution of the SchröDinger equation can be reduced to the problem of three harmonic oscillators. The frequencies of these oscillators were measured experimentally and thus the cyclotron frequency of free electrons was determined.
\end{abstract}

Um hochfrequenzspektroskopische Untersuchungen an freien Elektronen durchzuführen, müssen die Elektronen in einem gegebenen Volumen gespeichert werden. Hierzu eignet sich ein elektrischer Vierpolkäfig mit dem Potential

$$
V=\left(U_{0} / R^{2}\right)\left(x^{2}+y^{2}-2 z^{2}\right)
$$

und einem überlagerten homogenen Magnetfeld. In der vorliegenden Arbeit wird die Messung der Aufenthaltsdauer der Elektronen beschrieben. Anschließend wird die Lösung der SchröpIngerGleichung angegeben. Die Messung der Zyklotronfrequenzen der Elektronen zeigt vollständige Übereinstimmung mit den berechneten Energieniveaus.

\section{Apparatur}

Das elektrische Vierpolfeld wird realisiert durch zwei sich gegenüberstehende Pilzelektroden und einen in zwei Ringhälften geteilten Torus aus Platin-Iridium-Blechen (Abb. 1). Die Pilzelektroden bestehen aus zwei gegenüberliegenden Blechen, in die je ein Kugelsegment eingedrückt

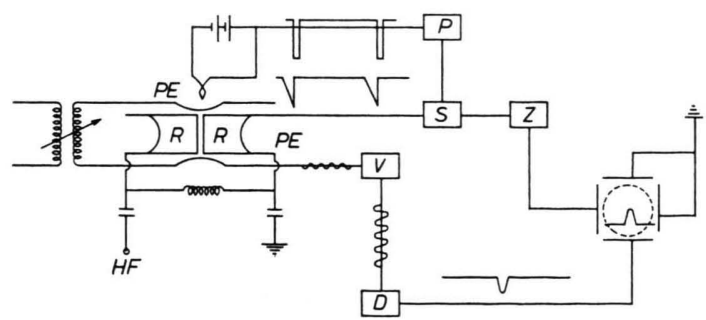

Abb. 1. Blockschaltbild. $\mathrm{P}=$ Pulsgenerator, $\mathrm{S}=$ Sägezahn, $\mathrm{Z}=$ Zeitgeber, $\mathrm{V}=$ Verstärker, $\mathrm{D}=$ Demodulation, $\mathrm{R}=$ Ringelektrode, $\mathrm{PE}=$ Pilzelektrode.

worden ist. Durch Anlegen einer Spannung $U_{0}$ zwischen den beiden Pilzelektroden einerseits und der Ringelektrode andererseits entsteht im Käfig ein Vierpolfeld der Form

$$
V(x, y, z)=\left(U_{0} / R^{2}\right)\left(x^{2}+y^{2}-2 z^{2}\right) .
$$

1 W. Paul, O. Osberghaus u. E. Fischer, Forschungsbericht des Wirtschaftsministeriums Nordrhein-Westfalen Nr. 415.
Dabei wurde die Symmetrieachse als $z$-Achse gewählt. Der innere Radius der Ringelektrode ist $R=0,8 \mathrm{~cm}$. Liegt das negative Potential an den Pilzelektroden, werden die Elektronen durch das elektrische Feld in $z$-Richtung stabilisiert. Am Verlassen des Käfigs in der Horizontalebene werden sie durch das überlagerte Magnetfeld in $z$-Richtung gehindert. Der Käfig ist an seinen elektrischen Zuführungen in einem Glasgehäuse aufgehängt, in dem mit Hilfe zweier Ionengetterpumpen ein Vakuum von einigen $10^{-10}$ Torr aufrechterhalten wird.

Abbildung 1 zeigt das Prinzip des Nachweises der Elektronen. Die positiv vorgespannte Kathode erhält zu Anfang einer Periode einen positiven Puls von 60 Volt, der die Elektronen in den Käfig schießt. Stabile Elektronen werden durch Ionisation des Restgases gebildet. Sie werden nachgewiesen über ihre harmonische Schwingung im elektrischen Parabelpotential des Käfigs in Magnetfeldrichtung. Die Frequenz $\omega_{0}$ dieser Schwingung ist nur abhängig von der Spannung und unabhängig vom Magnetfeld:

$$
\omega_{0}=\sqrt{4 e U_{0} / M R^{2}} .
$$

$M, e$ stellen Elektronenmasse und -ladung dar. Der Käfig ist Kapazität eines fremderregten Schwingkreises. Seine Schwingung wird in einem Schmalbandverstärker verstärkt, demoduliert und die Schwingungsamplitude auf die Vertikalablenkung des Oszillographen gegeben. Gleichzeitig mit der Zeitablenkung des Oszillographen fällt die Käfigspannung in einem Sägezahnabfall ab. Dabei wird nach Formel (1) die Spannung überfahren, bei der die Eigenfrequenz der Elektronenschwingung im Käfig übereinstimmt mit der Resonanzfrequenz des Schwingkreises. Beim Úberfahren der Resonanz absorbieren die Elektronen Energie aus dem Schwingkreis und die Amplitude seiner Schwingung wird gedämpft. Diese Dämpfung der Schwingungsamplitude ist auf dem Oszillographen sichtbar.

\section{Verweildauer}

Mit dieser Anordnung lassen sich Elektronen über eine Zeit von mehreren Minuten stabilisieren 1, 2, 3, 4. Abb. 2 zeigt das Elektronensignal und damit ein Maß für die Zahl der Elektronen in Abhängigkeit

2 E. Fischer, Z. Phys. 156, 1 (1959).

3 M. Barbier, CERN-Bericht $61-23$ [1961].

${ }^{4}$ H. W. Dahners, Diplomarbeit, Universität Bonn 1965. 
von der Zeit. Die Verweildauer der Elektronen im Käfig ist proportional dem Quadrat des angelegten Magnetfeldes und umgekehrt proportional dem im Käfig herrschenden Druck ${ }^{3,4}$.

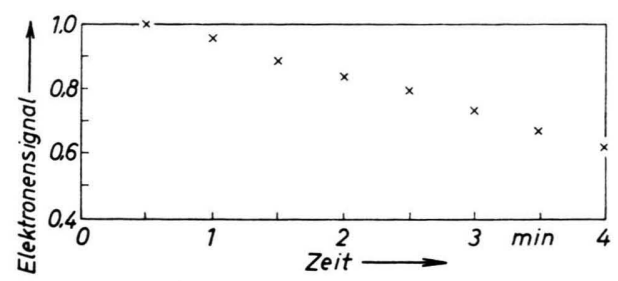

Abb. 2. Aufenthaltsdauer der Elektronen im Käfig. Es ist das Elektronensignal in willkürlichen Einheiten als Funktion der Zeit aufgetragen. $H=2500 \mathrm{GauB}, E=20 \mathrm{~V} / \mathrm{cm}$, $p=10^{-9}$ Torr.

\section{Messung der Zyklotronfrequenz}

\section{a) Theorie}

Der Hamilton-Operator eines nicht-relativistischen Elektrons in einem Magnetfeld $\boldsymbol{B}$ mit dem Vektorpotential $\boldsymbol{A}$ und einem elektrischen Feld $\boldsymbol{E}$ mit dem Potential $U$ ist gegeben durch ${ }^{5}$

$$
H=(1 / 2 M)(\boldsymbol{p}+e \boldsymbol{A})^{2}-e U ; \quad e>0 .
$$

Mit der Wahl des Vektorpotentials in der Form

$$
A=\frac{1}{2} B(-y, x, 0)
$$

läßt sich der Hamilton-Operator in eine Summe von drei harmonischen Oszillatoren aufspalten. Es ergibt sich als Gesamtenergie ${ }^{6}$

$$
E / \hbar=\left(n_{+}+\frac{1}{2}\right) \omega_{+}-\left(n_{-}+\frac{1}{2}\right) \omega_{-}+\left(n_{0}+\frac{1}{2}\right) \omega_{0} .
$$

In der $z$-Richtung haben wir die schon erwähnte harmonische Schwingung mit der Frequenz

$$
\omega_{0}=\sqrt{4 e U_{0} / M R^{2}} .
$$

Bei einer Käfigspannung von 10 Volt entspricht dies einer Frequenz von $40 \mathrm{MHz}$. In der $x-y$ Ebene haben wir einmal die gestörte Zyklotronfrequenz

$$
\omega_{+}=\frac{1}{2} \omega_{\mathrm{z}}+\sqrt{\frac{1}{4} \omega_{\mathrm{z}}^{2}-\frac{1}{2} \omega_{0}^{2}},
$$

die bei verschwindendem elektrischen Feld in die normale Zyklotronfrequenz

$$
\omega_{\mathrm{z}}=e \cdot B / M
$$

5 L. Landau, Z. Phys. 64, 629 [1930].

6 V. A. Duloch u. M. V. McIntosch, J. Math. Phys. 7, 1401 [1966]. übergeht. Außerdem tritt in den gekreuzten elektrischen und magnetischen Feldern eine Driftbewegung mit der Frequenz

auf.

$$
\omega_{-}=\frac{1}{2} \omega_{\mathrm{z}}-\sqrt{\frac{1}{4} \omega_{\mathrm{z}}^{2}-\frac{1}{2} \omega_{0}^{2}}
$$

Bei einem Magnetfeld von $100 \mathrm{G}$ erhält man als Zyklotronfrequenz $\omega_{+}=300 \mathrm{MHz}$, als Driftfrequenz $\omega_{-}=5 \mathrm{MHz}$. Da die Energie dieses harmonischen Oszillators mit höheren Quantenzahlen abnimmt, läßt sich die Driftfrequenz $\omega_{-}$auch in Emission beobachten.

Die Heisenberg-Gleichungen für die Bahnkurve des Elektrons lassen sich elementar lösen. Man erhält in $z$-Richtung eine harmonische Schwingung und in der Horizontalebene eine Epizykloide. Die die Epizykloide definierenden Radien sind mit dem Hamilton-Operator vertauschbar und damit Konstanten der Bewegung.

Relativistische Effekte äußern sich nur in einer Linienverschiebung

$$
(\Delta v) / v=E /\left(M c^{2}\right)
$$

und wegen der Energieverteilung der Elektronen in einer Linienverbreiterung ${ }^{7,8}$.

\section{b) Messungen}

Die Hochfrequenz wird zwischen den beiden Ringhälften eingestrahlt. Stimmt die eingestrahlte Frequenz überein mit einer der Eigenfrequenzen der Elektronen, werden die Elektronen aus dem Käfig geworfen, ihr Resonanzsignal auf dem Oszillographen verschwindet. Die erforderliche Amplitude für die Zentrallinie $\Delta n_{+}=1, \Delta n_{-}=\Delta n_{0}=0$ betrug rund $0,1 \mathrm{mV} / \mathrm{cm}$. Beobachtet wurden dabei die drei Grundfrequenzen des Elektrons $\omega_{0}, \omega_{+}, \omega_{-}$, d.h. die Frequenz der Schwingungen im elektrischen Potential des Käfigs, die Zyklotronfrequenz und die Frequenz der Drift der Elektronen um die Symmetrieachse des Käfigs, außerdem aber noch eine Vielzahl von Harmonischen dieser Frequenzen in der Form: $k \omega_{+}+n \omega_{-}+m \omega_{0} ; k, n, m=0, \pm 1$, $\pm 2, \ldots$, ganzzahlig. Die Messungen wurden im Bereich von $80-4000 \mathrm{Gau}$ und von $5-20 \mathrm{~V} / \mathrm{cm}$ durchgeführt. Abb. 3 zeigt das Spektrum der Frequenzen, bei denen die Elektronen den Käfig verlassen, in Abhängigkeit von der Käfigspannung. Da

7 M. H. Johnson u. B. A. Lippmann, Phys. Rev. 76, 828 [1949].

8 R. Honeruäger u. E. Klein, Z. Phys. 169, 32 [1962]. 
die Driftfrequenz $\omega_{-}$proportional der Käfigspannung ist und die Frequenz der Schwingung im elektrischen Potential $\omega_{0}$ proportional der Wurzel aus der Käfigspannung, erhält man entsprechend der Lösung der ScHRÖDINGER-Gleichung eine große parabelförmige und eine schwächere lineare Aufspaltung der Zyklotronfrequenz. Wegen der großen Zahl

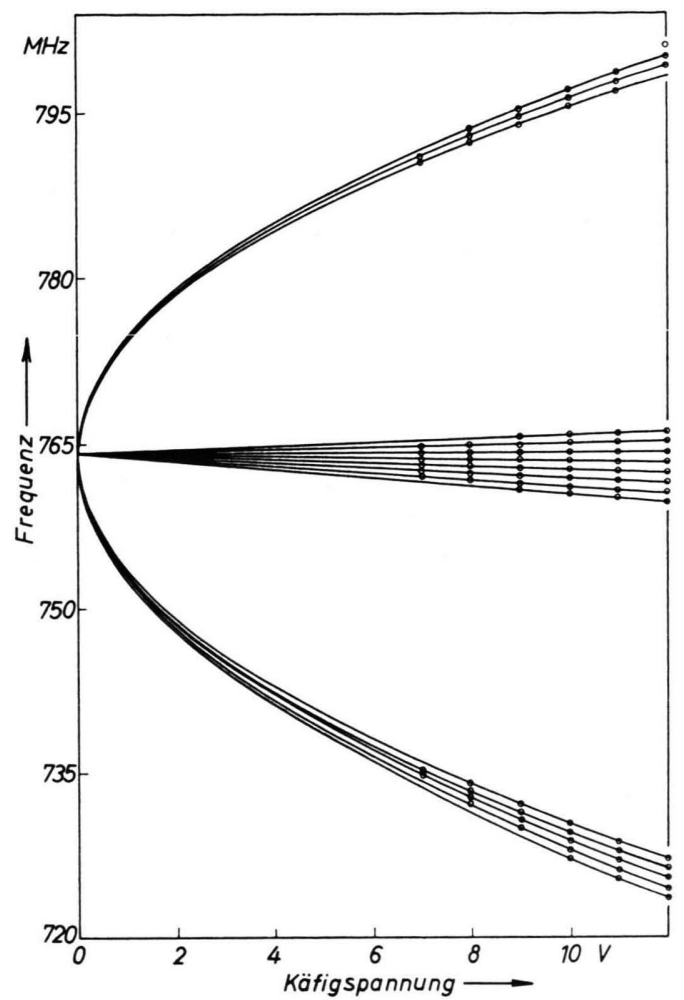

Abb. 3. Frequenzspektrum in Abhängigkeit von der Käfigspannung. Die Meßpunkte sind durch angepaßte Geraden bzw. Parabeln miteinander verbunden. der Linien läßt sich die Zyklotronfrequenz bei verschwindendem elektrischem Feld mit großer Genauigkeit extrapolieren. Die Messung des Magnetfeldes ist also zurückgeführt auf eine Messung von Frequenzen.

Ungeklärt ist bisher die Frage der Linienbreite. Auf Grund relativistischer Effekte sollten die Linienbreite und die erforderlichen Hochfrequenzamplituden erheblich größer sein als die gemessenen. Die Linienbreite der Zentrallinie beträgt annähernd unabhängig von der Magnetfeldstärke rund $20 \mathrm{kHz}$. Bei höheren Magnetfeldfeldern (3000 Gauß) konnte folglich eine relative Linienbreite von fast $10^{-6} \mathrm{er}$ reicht werden.

Die Linienbreiten können erklärt werden, wenn man annimmt, daß die Energie der Elektronen sich schon während der Einstrahlung der HF auf die drei Freiheitsgrade des Elektrons verteilt. Das Elektron kann dann den Käfig schon bei einem Energiegewinn von etwa $2 \mathrm{eV}$ verlassen. Dem entspricht eine Linienbreite von $4 \cdot 10^{-6}$. Wir werden versuchen, diese Frage durch weitere Untersuchungen zu klären*.

Herrn Prof. Dr. W. Paul sind wir für sein Interesse an dieser Arbeit und viele Dikussionen zu großem Dank verpflichtet. Wir danken Herrn Dipl. Phys. Roeder und Herrn Dipl. Phys. Werth für ihre Hilfe beim Aufbau der Apparatur, Herrn Dipl. Phys. Dahners für seine Hilfe bei einem Teil der Messungen. - Der Deutschen Forschungsgemeinschaft danken wir für die Utberlassung vieler Geräte, die die Durchführung der Messung ermöglichten.

* Anm. b. d. Korr.: Wie wir während des Druckes dieser Arbeit erfuhren, wurden die gleichen Rechnungen von A. A. Sokolov und Ju. G. Pavlenko in Optics and Spectroscopy XXII, l veröffentlicht. 